\title{
Gemcitabine maintenance versus observation after first-line chemotherapy in patients with metastatic urothelial carcinoma: a retrospective study
}

\author{
Hyunho Kim ${ }^{1}$, Seung-Hwan Lee ${ }^{2}$, Dong Hwan Kim ${ }^{3}$, Ji Youl Lee ${ }^{4,5}$, Sung-Hoo Hong ${ }^{4,5}$, U-Syn Ha ${ }^{4,5}$, \\ In-Ho Kim ${ }^{2,5,6}$
}

${ }^{1}$ Division of Medical Oncology, Department of Internal Medicine, The Catholic University of Korea, St. Vincent's Hospital, Suwon, Republic of Korea; ${ }^{2}$ Division of Medical Oncology, Department of Internal Medicine, The Catholic University of Korea, Seoul St. Mary's Hospital, Seoul, Republic of Korea; ${ }^{3}$ Department of Radiology, The Catholic University of Korea, Seoul St. Mary's Hospital, Seoul, Republic of Korea; ${ }^{4}$ Department of Urology Cancer Center, The Catholic University of Korea, Seoul St. Mary's Hospital, Seoul, Republic of Korea; ${ }^{5}$ Department of Genitourinary Cancer Centre, Seoul St. Mary's Hospital, College of Medicine, The Catholic University of Korea, Seoul, Republic of Korea; ${ }^{6}$ Cancer Research Institute, College of Medicine, The Catholic University of Korea, Seoul, Republic of Korea

Contributions: (I) Conception and design: H Kim, IH Kim; (II) Administrative support: H Kim, SH Lee; (III) Provision of study materials or patients: JY Lee, SH Hong, US Ha, IH Kim; (IV) Collection and assembly of data: H Kim, SH Lee, DH Kim, IH Kim; (V) Data analysis and interpretation: H Kim, IH Kim; (VI) Manuscript writing: All authors; (VII) Final approval of manuscript: All authors.

Correspondence to: In-Ho Kim, MD, PhD. Department of Internal Medicine, Seoul St. Mary's Hospital, The Catholic University of Korea College of Medicine, 222 Banpo-daero, Seocho-gu, Seoul 137-701, Republic of Korea. Email: ihkmd@catholic.ac.kr.

Background: Gemcitabine with platinum is one of the most important first-line treatments for metastatic urothelial cancer (mUC). However, continuation of platinum agents results in cumulative toxicities, such as nephrotoxicity, ototoxicity, and neurotoxicity, which lead to discontinuation of chemotherapy after 4-6 cycles despite a favorable response in the patients. The strategy of maintenance treatment can give clinical benefit to patients, but there is no consensus about maintenance treatment. The aim of this study was to investigate the clinical impact of the gemcitabine maintenance (GEM-m) in mUC patients who achieve disease control from first-line gemcitabine with platinum agents.

Methods: A total of 117 patients who showed response to 4-6 cycles of gemcitabine plus cisplatin or carboplatin as the first-line palliative chemotherapy were reviewed between 2014 to 2018. Patients who were treated with GEM-m received a $1,000 \mathrm{mg} / \mathrm{m}^{2}$ dose of gemcitabine on day 1 and 8 for 3 weeks until disease progression or development of unacceptable toxicity. The patients who are not treated with GEM-m were followed up with regular radiologic evaluation. Statistical analyses were performed using the log-rank test and Cox proportional hazards method.

Results: Fifty-eight patients (49.6\%) received GEM-m. The median cycle of GEM-m was 4 (range, 1-12). Six patients (10.3\%) in the GEM-m group showed an objective response. A median overall survival (OS) of 11.8 months and 9.6 months was observed for the GEM-m and non-GEM-m groups, respectively [HR 0.621; 95\% CI, 0.39-0.97; P=0.026]. Additionally, median progression-free survival (PFS) was 4.6 months and 3.3 months in the GEM-m and non-GEM-m groups, respectively [HR 0.612; 95\% CI, 0.41-0.91; P=0.009]. Grade 3 or higher neutropenia occurred in $17.2 \%$ of patients in the GEM-m and $1.7 \%$ in the non-GEM-m group.

Conclusions: Our results suggest that GEM-m can be considered in patients who respond to gemcitabine with platinum. Large-scale prospective study should be warranted.

Keywords: Maintenance chemotherapy; survival; transitional cell carcinoma

Submitted Mar 18, 2020. Accepted for publication Sep 11, 2020.

doi: 10.21037/tau-20-772

View this article at: http://dx.doi.org/10.21037/tau-20-772 


\section{Introduction}

Bladder cancer is the $9^{\text {th }}$ most common cancer worldwide and the $7^{\text {th }}$ most common among men (1). About 430,000 new cases were reported with 165,000 deaths in the year 2012 (2). Urothelial carcinoma is the most common histologic subtype and accounts for $90-95 \%$ cases of bladder cancer (3). Main treatment for patients with inoperable locally advanced or metastatic urothelial cancer (mUC) comprises of systemic chemotherapy, and gemcitabine plus cisplatin (GP) is the most widely used in first-line setting in these patients (4). Compared to MVAC (methotrexate, vinblastine, doxorubicin, and cisplatin), which was previous standard $1^{\text {st }}$ line chemotherapy for mUC, GP showed similar survival, better safety profile, and tolerability (5-7). In patients who are unfit for cisplatin, gemcitabine with carboplatin is most widely used, which provides about $40 \%$ response rate with comparable survival outcomes $(8,9)$. Long-term treatment with platinum results in decreased performance status and several toxicities, such as nephrotoxicity, peripheral neuropathy, and irreversible hearing loss, and hence results in its discontinuation $(10,11)$. Moreover, the incidence of these side effects increase with the number and cumulative dose of chemotherapy cycles. Thus, treatment for mUC mostly consists of 4-6 cycles of platinum-based chemotherapy, and most patients experience disease-progression after discontinuing chemotherapy (12). Recently, the immune checkpoint inhibitors (ICIs), such as atezolizumab, pembrolizumab, nivolumab, durvalumab, and avelumab, have showed clinical benefit in second or above settings (13). Despite the availability of these promising agents, platinum-based chemotherapy is still a mainstay of first-line treatment in mUC. Therefore, there is an unmet need to maximize the therapeutic effect of firstline chemotherapy. Previous studies suggested utility of maintenance approach after first-line treatment in patients who respond to first line treatment $(14,15)$. However, due to the small number of patients (17 subjects) or regimen consisting of 5 -FU based chemotherapy $(14,15)$, further studies are needed for patients treated with Gemcitabine with platinum.

Gemcitabine, a difluorinated deoxycytidine analogue, enters the cancer cells and transforms into an active triphosphate metabolite (16). This active form interferes with DNA synthesis and DNA repair processes, and thus imparting its antitumor effects (16). Gemcitabine monotherapy provides a $25 \%$ overall response rate and presents with tolerable toxicity $(12,17)$, such as the commonly occurring myelosuppression, in patients with mUC $(16,18)$. These previous studies on gemcitabine monotherapy raise the expectation of gemcitabine maintenance (GEM-m) for mUC to avoid the cumulative toxicity caused by platinum and to maintain the therapeutic effect.

Based on these considerations, we investigated the clinical implication of GEM-m in patients who achieve disease control after first-line gemcitabine with platinum chemotherapy, retrospectively. We present the following article in accordance with the STROBE reporting checklist (available at http://dx.doi.org/10.21037/tau-20-772).

\section{Methods}

\section{Study population}

All the patients who were included in this study had locally advanced and unresectable or metastatic urothelial carcinoma. Additionally, this study included patients who were treated with gemcitabine plus platinum (cisplatin or carboplatin) as 1st line chemotherapy and 0-2 of ECOG performance status. The medical records of 117 patients, who presented with radiological response [complete response (CR), partial response (PR), and stable disease (SD) by Response Evaluation Criteria in Solid Tumors (RECIST) version 1.1] following 4-6 cycles of first-line gemcitabine with, were reviewed (Figure 1).

The study was conducted in accordance with the Declaration of Helsinki (as revised in 2013) and the Harmonized Tripartite Guideline for Good Clinical Practice from the International Conference on Harmonization. This retrospective study was approved by the Institutional Review Board of the Seoul St. Mary's Hospital of the Catholic University of Korea (KC20RISI0160). Further, informed consent was not required as we only reviewed their routine medical records.

\section{Treatment and response evaluation}

Patients were treated with 4-6 cycles of gemcitabine plus cisplatin or carboplatin as a first-line palliative chemotherapy between the years 2012 and 2018. This included intravenous administration of $1,000 \mathrm{mg} / \mathrm{m}^{2}$ gemcitabine on days 1 and 8 , while $70 \mathrm{mg} / \mathrm{m}^{2}$ cisplatin or 5/AUC carboplatin on day 1 , every 3 weeks. Among the responders (CR, PR and $\mathrm{SD}$ ) to gemcitabine with platinum, GEM-m or observation without GEM-m (non-GEM-m) 


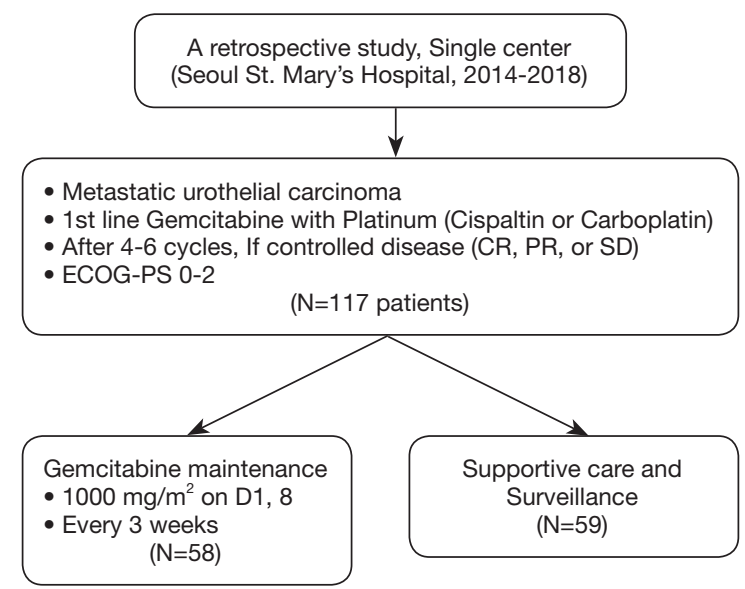

Figure 1 Study design.

were performed to these patients. GEM-m or nonGEM- $m$ was determined in consideration of the clinical factors of patients and clinician's decision (intolerability to chemotherapy, patient's denial of GEM-m, and so on) The GEM-m group received $1,000 \mathrm{mg} / \mathrm{m}^{2}$ gemcitabine, on days 1 and 8 every 3 weeks, until disease progression or unacceptable toxicity. Non-GEM-m was continued until disease progression. Baseline imaging with computed tomography (CT) imaging of the chest, abdomen, and pelvis was acquired within 4 weeks after completion of the first-line chemotherapy. Both GEM-m and non-GEM-m group received regular radiographic evaluation with CT scan every $6 \pm 2$ weeks. Radiological changes were evaluated using the RECIST version 1.1. Hematologic toxicities were graded according to National Cancer Institute Common Toxicity Criteria for Adverse Events version 5.0.

\section{Statistical analysis}

Overall survival (OS) and progression-free survival (PFS) were defined as the time from baseline imaging date till death from any cause and disease progression or death, respectively. The baseline characteristics were compared using chi-squared or Fisher's exact test for category. Survival curves were obtained using the Kaplan-Meier method, and compared using the log-rank test. The Cox proportional hazards method was used to perform multivariate analysis to evaluate the significance of the prognostic variables for PFS and OS. All statistical analyses were performed using SPSS software (version 24; IBM Corp., Armonk, NY, USA), and a two-sided $\mathrm{P}$ value $<0.05$ was considered statistically significant.

\section{Results}

\section{Baseline characteristics}

A total of 117 patients were included in this study; 58 patients (49.6\%) received GEM-m. Distribution of clinical factors including response to first-line chemotherapy were not different between GEM-m and non-GEM-m. The baseline characteristics of both the patient groups have been summarized in Table 1. The risk factors included Hemoglobin $<10 \mathrm{~g} / \mathrm{dL}$, ECOG performance status score of 1 or above and presence of liver metastases (19).

\section{Response rate and Survival outcome in mUC patients treated with GEM-m}

GEM-m was administered with a median of 4 cycles (range, 1-12 cycles) until disease progression or unacceptable toxicity. Disease progression was the most common cause for GEM-m discontinuation (55\%). An objective response was observed in 6 patients $(10.3 \%)$, while two patients showed CR (Table 2). Median OS were 11.8 and 9.6 months in patients treated with GEM-m and non-GEM-m, respectively [hazard ratio (HR), 0.621; 95\% confidence interval (CI), 0.39-0.97; $\mathrm{P}=0.026]$. Moreover, the median PFS were 4.6 vs. 3.3 months in the GEM-m and nonGEM-m, respectively (HR 0.612; 95\% CI, 0.41-0.91; $\mathrm{P}=0.009$ ) (Figure 2). The Multivariate analysis using Cox regression suggested significant association of GEM-m and the 2 or higher risk factors with OS and PFS (Figure 3). Further, after disease progression, second-line treatment was administered to $35(60.3 \%)$ and 15 patients $(25.4 \%)$ in the GEM-m and non-GEM-m, respectively. 
Table 1 Baseline characteristics

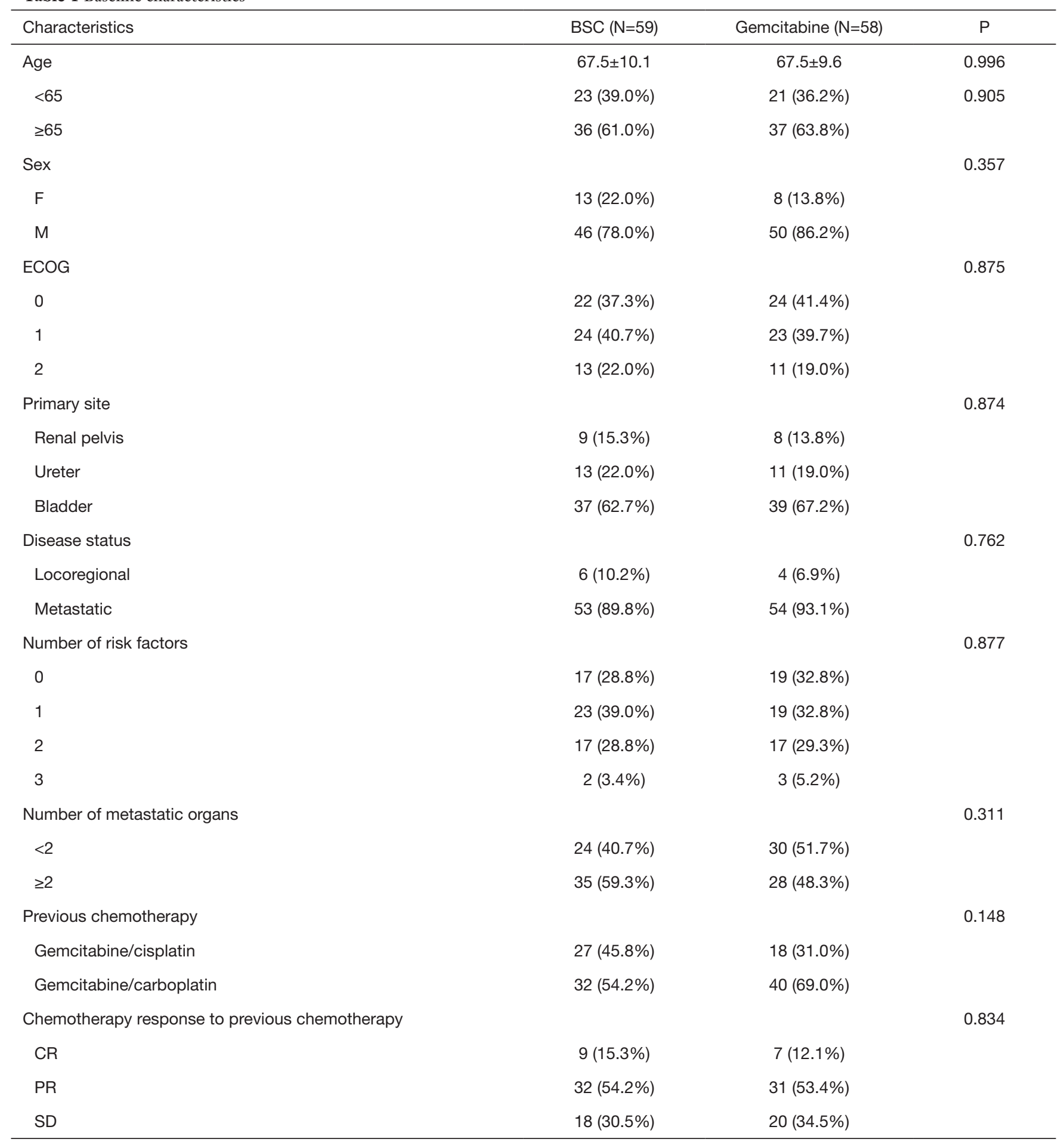

Risk factors: hemoglobin $<10 \mathrm{~g} / \mathrm{dL}$, ECOG performance status score of 1 or above, presence of liver metastases. BSC, best supportive care; CR, complete response; ECOG, eastern cooperative oncology group; PR, partial response; SD, stable disease. 
Table 2 Treatment response

\begin{tabular}{lccccc}
\hline Variables & CR & PR & SD & PD & Total \\
\hline BSC & $0(0.0 \%)$ & $0(0.0 \%)$ & $39(66.1 \%)$ & $20(33.9 \%)$ & $59(100 \%)$ \\
Gemcitabine & $2(3.4 \%)$ & $4(6.9 \%)$ & $35(60.3 \%)$ & $17(29.3 \%)$ & $58(100 \%)$ \\
Total & $2(1.7 \%)$ & $4(3.4 \%)$ & $74(63.2 \%)$ & $37(31.7 \%)$ & $117(100 \%)$ \\
\hline
\end{tabular}

BSC, best supportive care; CR, complete response; PR, partial response; PD, progressive disease; SD, stable disease.
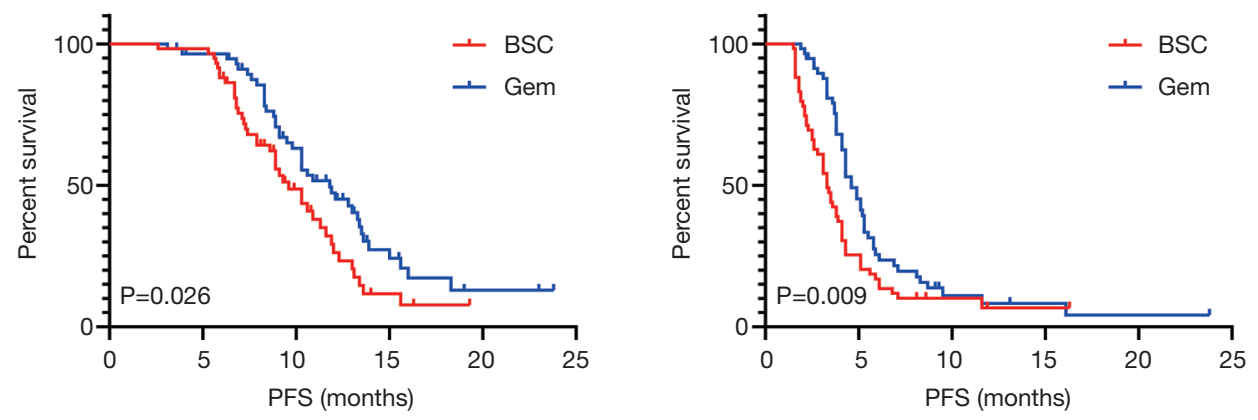

Figure 2 Survival curves by using the Kaplan-Meier method. BSC, best supportive care; Gem, gemcitabine maintenance; OS, overall survival; PFS, progression free survival.
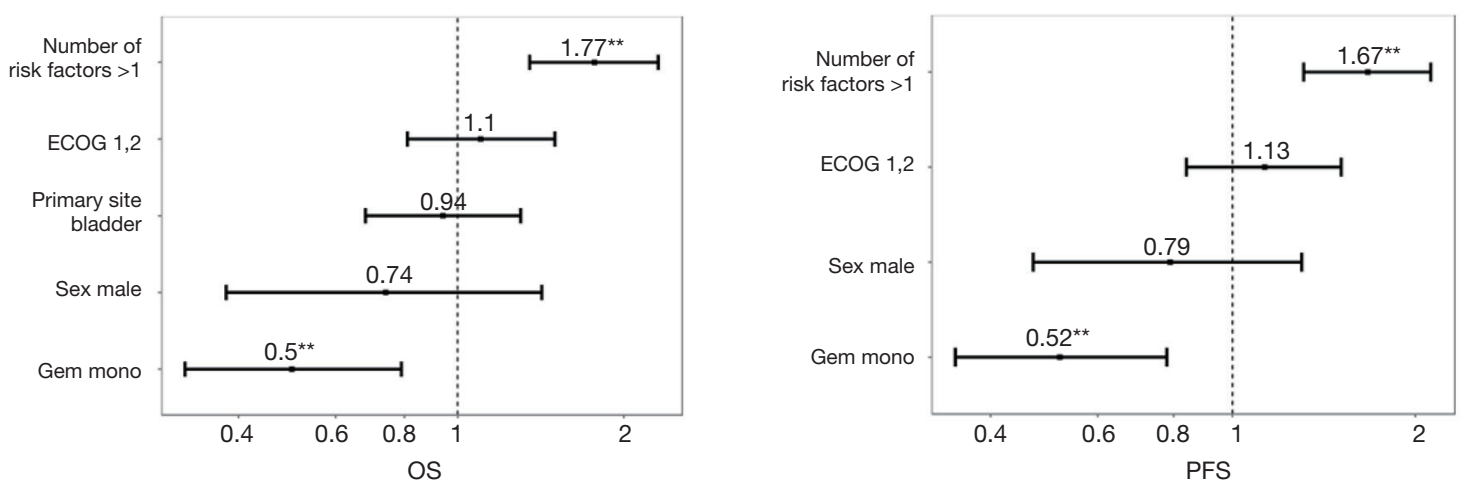

Figure 3 Multivariate analysis with cox regression for survival. Risk factor: Hemoglobin $<10 \mathrm{~g} / \mathrm{dL}$, ECOG performance status score of 1 or above, presence of liver metastasis. OS, overall survival; PFS, progression free survival. ${ }^{* *}$ means that there are statistically significant.

\section{Safety associated with hematologic toxicities}

Neutropenia was the most common hematologic toxicity, and $10(17.2 \%), 8(13.8 \%), 9(15.5 \%)$, and $1(1.7 \%)$ patient in the GEM-m presented with grade $1,2,3$, and 4 neutropenia, respectively. Febrile neutropenia was observed in 2 patients during GEM-m, while the other hematologic toxicities were similar in the GEM-m and non-GEM-m. Although the risk of neutropenia was significantly higher in
GEM-m, GEM-m was tolerable as a maintenance treatment after first-line gemcitabine with platinum chemotherapy (Table 3).

\section{Discussion}

There is a strong correlation between PFS and OS in solid cancers, although it may not be always consistent $(20,21)$. Therefore, efforts are being taken to prolong the duration 
Table 3 Hematologic toxicities

\begin{tabular}{|c|c|c|c|}
\hline Toxicity (grade) & $\mathrm{BSC}(\mathrm{N}=59)$ & Gemcitabine $(\mathrm{N}=58)$ & $\mathrm{P}$ \\
\hline Neutropenia & & & 0.013 \\
\hline 0 & 45 (76.3\%) & $30(51.7 \%)$ & \\
\hline 1 & 10 (16.9\%) & $10(17.2 \%)$ & \\
\hline 2 & $3(5.1 \%)$ & $8(13.8 \%)$ & \\
\hline 3 & $1(1.7 \%)$ & $9(15.5 \%)$ & \\
\hline 4 & $0(0.0 \%)$ & $1(1.7 \%)$ & \\
\hline Anemia & & & 0.167 \\
\hline 0 & 47 (79.7\%) & $39(67.2 \%)$ & \\
\hline 1 & $10(16.9 \%)$ & $11(19.0 \%)$ & \\
\hline 2 & $2(3.4 \%)$ & $5(8.6 \%)$ & \\
\hline 3 & $0(0.0 \%)$ & $3(5.2 \%)$ & \\
\hline Thrombocytopenia & & & 0.325 \\
\hline 0 & 55 (93.2\%) & $48(82.8 \%)$ & \\
\hline 1 & $3(5.1 \%)$ & $6(10.3 \%)$ & \\
\hline 2 & $1(1.7 \%)$ & $3(5.2 \%)$ & \\
\hline 3 & $0(0.0 \%)$ & $1(1.7 \%)$ & \\
\hline Febrile neutropenia & & & 0.988 \\
\hline No & 58 (98.3\%) & $56(96.6 \%)$ & \\
\hline Yes & $1(1.7 \%)$ & $2(3.4 \%)$ & \\
\hline
\end{tabular}

of treatment response in the first-line chemotherapy responders. This is especially important for the mUC patients that show short PFS of 6-7 months upon GP chemotherapy $(5,7,12)$, and maintenance treatment could be one of such efforts. The rationale behind the treatment is to increase exposure to effective therapies, decrease resistance to chemotherapy, and maximize the antitumor efficacy (21). We ultimately expect that chemotherapy maintenance can preserve the chances of subsequent therapy opportunities arising from prevention of abrupt deterioration, as well as enable long-term disease control.

This study showed improvement in survival of patients, although with modest benefit. The modest 1.3 and 2.2 months' improvement in PFS and OS, respectively, is rather difficult to interpret considering the quality of life (QOL) and cost effective. The chemotherapy-free interval following first-line regimen is the time taken to recover from toxicities of platinum agents and is usually a period of disease-related symptom control. Maintenance chemotherapy during this period would lead to about $30 \%$ of patients to continue and worsen general weakness and fatigue, and experience a decline in QOL (22). Whereas, it may also be possible to maintain or improve QOL while the disease is under control. This notion is supported by a pancreatic cancer study, which showed an improvement in QOL during the duration of the chemotherapy, and no worsening of QOL until one month before treatment failure $(23,24)$. Further, continued disease control could slow the rate of deterioration of QOL $(23,24)$. Thus, it is difficult to determine the balance between maintenance and rest of the chemotherapeutic regimen. Several preference studies have been published in this regard for NSCLC chemotherapy (25-27). First, the median OS benefit threshold, where the patients received chemotherapy despite the risks including a drop in QOL and side effects, was 4.5 months, and $70 \%$ of the patients chose best supportive care when they showed a three month survival benefit $(21,27)$. The PFS benefits were worth treating if the patients had mild 
adverse events, though, many of the patients did not opt for intense chemotherapy $(21,25)$. However, in a different survey, $43 \%$ of the patients assumed that they would receive chemotherapy with only one month survival benefit (26), which suggests that despite the smaller effects, certain patients willingly opt for the chemotherapy maintenance. In terms of cost of the treatment per patient, the treatment with gemcitabine does not require hydration, and can be administered in an outpatient clinic, which helps maintain QOL and also benefits in terms of cost savings.

The treatment with gemcitabine is actively followed for mUC (12), and similar outcomes and mild toxicity have been obtained in patients above and below 65 years of age $(16,28)$. This observation suggests that gemcitabine may help in treating relatively elderly patients with mUC. The decreased renal function, together with mUC at origin of urinary tract, nephrectomy or old age, influence the consideration of repetitive chemotherapy. Moreover, gemcitabine is primarily metabolized in the liver, instead of kidney, and is therefore suitable for long-term dosing (18). Indeed, as reported by Kuş et al., treatment of patients with GEM-m after 6 cycles of gemcitabine with platinum $(\mathrm{N}=23)$ conferred a survival benefit, and showed grade 3 hematotoxicity in $30.4 \%$ cases (28). Further, gemcitabine monotherapy has shown favorable results in terms of benefits and side effects in other similar studies $(17,18)$. Akaza et al. reported that second-line gemcitabine treatment in patients progressing after MVAC showed median PFS of 3.1 months, grade $3 / 4$ bone marrow suppression in $47.7 \%$ cases, and grade $3 / 4$ non-hematologic toxicities in $9.1 \%$ cases (17). Another study has compared the outcomes upon treatment with GEM-m following platinum based chemotherapy versus best supportive care (33 patients each), which suggested differences in time to progression (12 vs. 2 months) in both these groups, together with grad $3 / 4$ hematologic and non-hematologic toxicities upon GEM-m as $27.3 \%$ and 3\%, respectively (18). Moreover, our study showed a similar survival benefit and grade $3 / 4$ hematologic toxicity in $27.5 \%$ cases. Taken together, these results suggest that treatment with GEM-m is beneficial with manageable toxicities in patients with $\operatorname{mUC}(17,18,28)$.

However, our study has few limitations. First, owing to the retrospective nature of the study, selection bias and issues with accuracy of data collected are inevitable. Second, although hematologic toxicities were assessed, we did not include data related to QOL, such as pain, fatigue, and other subjective factors, which affects the evaluation of comprehensive palliative effects (24). Moreover, survival benefits need to be interpreted with care due to smaller number of patients reviewed, which warrants identification of subgroups that could benefit from GEM given the low survival benefit obtained in our study. Furthermore, there are no prospective studies yet that have analyzed the effect of GEM-m on mUC. Additionally, similar to bladder cancer, other cancer types have not been studied with regard to the effectiveness of maintenance therapy, making them ineligible as a reference $(21,29)$. Therefore, well-designed prospective studies enrolling large number of patients, with QOL and subgroup analysis, would be needed to support the results of this study.

Taken together, it is important to stratify appropriate patients because GEM may not benefit or apply to all patients treated with 6 cycles of gemcitabine with platinum. Additionally, factors expected to benefit from maintenance treatment include performance status, response to first-line therapy, and compliance to subsequent treatment related to disease-burden and socioeconomic status (21). We suggest that GEM-M can be considered for selected patients who achieve disease control from $1^{\text {st }}$ line gemcitabine with platinum.

\section{Acknowledgments}

Funding: None.

\section{Footnote}

Reporting Checklist: The authors have completed the STROBE reporting checklist. Available at http://dx.doi. org/10.21037/tau-20-772

Data Sharing Statement: Available at http://dx.doi. org/10.21037/tau-20-772

Conflicts of Interest: All authors have completed the ICMJE uniform disclosure form (available at http://dx.doi. org/10.21037/tau-20-772). The authors have no conflicts of interest to declare.

Ethical Statement: The authors are accountable for all aspects of the work in ensuring that questions related to the accuracy or integrity of any part of the work are appropriately investigated and resolved. The study was conducted in accordance with the Declaration of Helsinki (as revised in 2013) and the Harmonized Tripartite Guideline for Good Clinical Practice from the International 
Conference on Harmonization. The retrospective study was approved by the IRB of the Seoul St. Mary's Hospital of the Catholic University of Korea (KC20RISI0160). The need for informed consent was waived by the IRB of the Seoul St. Mary's Hospital of the Catholic University of Korea due to the study design as this was a retrospective review of medical records.

Open Access Statement: This is an Open Access article distributed in accordance with the Creative Commons Attribution-NonCommercial-NoDerivs 4.0 International License (CC BY-NC-ND 4.0), which permits the noncommercial replication and distribution of the article with the strict proviso that no changes or edits are made and the original work is properly cited (including links to both the formal publication through the relevant DOI and the license). See: https://creativecommons.org/licenses/by-nc-nd/4.0/.

\section{References}

1. Cumberbatch MGK, Noon AP. Epidemiology, aetiology and screening of bladder cancer. Transl Androl Urol 2019;8:5-11.

2. Antoni S, Ferlay J, Soerjomataram I, et al. Bladder Cancer Incidence and Mortality: A Global Overview and Recent Trends. Eur Urol 2017;71:96-108.

3. Reuter VE. The pathology of bladder cancer. Urology 2006;67:11-7; discussion 17-8.

4. Moore MJ, Winquist EW, Murray N, et al. Gemcitabine plus cisplatin, an active regimen in advanced urothelial cancer: a phase II trial of the National Cancer Institute of Canada Clinical Trials Group. J Clin Oncol 1999;17:2876-81.

5. Roberts JT, von der Maase H, Sengelov L, et al. Longterm survival results of a randomized trial comparing gemcitabine/cisplatin and methotrexate/vinblastine/ doxorubicin/cisplatin in patients with locally advanced and metastatic bladder cancer. Ann Oncol 2006;17 Suppl 5:v118-22.

6. Sternberg CN, Yagoda A, Scher HI, et al. M-VAC (methotrexate, vinblastine, doxorubicin and cisplatin) for advanced transitional cell carcinoma of the urothelium. J Urol 1988;139:461-9.

7. von der Maase H, Sengelov L, Roberts JT, et al. Longterm survival results of a randomized trial comparing gemcitabine plus cisplatin, with methotrexate, vinblastine, doxorubicin, plus cisplatin in patients with bladder cancer. J Clin Oncol 2005;23:4602-8.
8. De Santis M, Bellmunt J, Mead G, et al. Randomized phase II/III trial assessing gemcitabine/carboplatin and methotrexate/carboplatin/vinblastine in patients with advanced urothelial cancer who are unfit for cisplatinbased chemotherapy: EORTC study 30986. J Clin Oncol 2012;30:191-9.

9. Dogliotti L, Carteni G, Siena S, et al. Gemcitabine plus cisplatin versus gemcitabine plus carboplatin as first-line chemotherapy in advanced transitional cell carcinoma of the urothelium: results of a randomized phase 2 trial. Eur Urol 2007;52:134-41.

10. Dasari S, Tchounwou PB. Cisplatin in cancer therapy: molecular mechanisms of action. Eur J Pharmacol 2014;740:364-78.

11. Oun R, Moussa YE, Wheate NJ. The side effects of platinum-based chemotherapy drugs: a review for chemists. Dalton Trans 2018;47:6645-53.

12. Shelley MD, Cleves A, Wilt TJ, et al. Gemcitabine chemotherapy for the treatment of metastatic bladder carcinoma. BJU Int 2011;108:168-79.

13. Hsu FS, Su CH, Huang KH. A Comprehensive Review of US FDA-Approved Immune Checkpoint Inhibitors in Urothelial Carcinoma. J Immunol Res 2017;2017:6940546.

14. Liaw CC, Liao TY, Tsui KH, et al. Survival Benefit for Patients With Metastatic Urothelial Carcinoma Receiving Continuous Maintenance Chemotherapy. In Vivo 2019;33:1249-62.

15. Mitsuzuka K, Yamashita S, Namiki S, et al. Low-dose maintenance gemcitabine-carboplatin chemotherapy could be an alternative to continuous standard chemotherapy for patients with metastatic urothelial carcinoma. Int J Urol 2014;21:1114-9.

16. Tonato M, Mosconi AM, Martin C. Safety profile of gemcitabine. Anticancer Drugs 1995;6 Suppl 6:27-32.

17. Akaza H, Naito S, Usami M, et al. Efficacy and Safety of Gemcitabine Monotherapy in Patients with Transitional Cell Carcinoma after Cisplatin-Containing Therapy: A Japanese Experience. Jpn J Clin Oncol 2007;37:201-6.

18. Muto S, Abe H, Noguchi T, et al. Maintenance monotherapy with gemcitabine after standard platinumbased chemotherapy in patients with advanced urothelial cancer. Int J Urol 2015;22:490-4.

19. Garcia-Donas J, Font A, Perez-Valderrama B, et al. Maintenance therapy with vinflunine plus best supportive care versus best supportive care alone in patients with advanced urothelial carcinoma with a response after first-line chemotherapy (MAJA; SOGUG 2011/02): a multicentre, randomised, controlled, open-label, phase 2 
trial. Lancet Oncol 2017;18:672-81a.

20. Davis S, Tappenden P, Cantrell A. NICE Decision Support Unit Methods Development. A Review of Studies Examining the Relationship between Progression-Free Survival and Overall Survival in Advanced or Metastatic Cancer. London: National Institute for Health and Care Excellence (NICE). All rights reserved; 2012.

21. Gerber DE, Schiller JH. Maintenance chemotherapy for advanced non-small-cell lung cancer: new life for an old idea. J Clin Oncol 2013;31:1009.

22. Iop A, Manfredi AM, Bonura S. Fatigue in cancer patients receiving chemotherapy: an analysis of published studies. Ann Oncol 2004;15:712-20.

23. Bernhard J, Dietrich D, Scheithauer W, et al. Clinical benefit and quality of life in patients with advanced pancreatic cancer receiving gemcitabine plus capecitabine versus gemcitabine alone: a randomized multicenter phase III clinical trial--SAKK 44/00-CECOG/PAN.1.3.001. J Clin Oncol 2008;26:3695-701.

24. Reni M, Bonetto E, Cordio S, et al. Quality of life

Cite this article as: $\mathrm{Kim} \mathrm{H}$, Lee $\mathrm{SH}$, Kim DH, Lee JY, Hong SH, Ha US, Kim IH. Gemcitabine maintenance versus observation after first-line chemotherapy in patients with metastatic urothelial carcinoma: a retrospective study. Transl Androl Urol 2020;9(5):2113-2121. doi:10.21037/tau-20-772 assessment in advanced pancreatic adenocarcinoma: results from a phase III randomized trial. Pancreatology 2006;6:454-63.

25. Bridges JF, Mohamed AF, Finnern HW, et al. Patients' preferences for treatment outcomes for advanced nonsmall cell lung cancer: a conjoint analysis. Lung Cancer 2012;77:224-31.

26. Peeters L, Sibille A, Anrys B, et al. Maintenance therapy for advanced non-small-cell lung cancer: a pilot study on patients' perceptions. J Thorac Oncol 2012;7:1291-5.

27. Silvestri G, Pritchard R, Welch HG. Preferences for chemotherapy in patients with advanced non-small cell lung cancer: descriptive study based on scripted interviews. BMJ 1998;317:771-5.

28. Kuş T, Aktaş G. Maintenance treatment with gemcitabine have a promising activity on metastatic bladder cancer survival. Turk J Urol 2017;43:273.

29. Giuliani F, De Vita F, Colucci G, et al. Maintenance therapy in colon cancer. Cancer Treat Rev 2010;36 Suppl $3: S 42-5$. 